\title{
Article
}

\section{Design of a Quasi Direct drive Actuator for Dynamic Motions}

\author{
Akash Singh $^{1,2}$, Navvab Kashiri ${ }^{1}$ and Nikolaos Tsagarakis ${ }^{1}$ \\ 1 Istituto Italiano di Tecnologia, Genoa, Italy; \\ 2 University of Genoa, Italy \\ * Corresponding author's email: Akash.singh@iit.it \\ + Current address: Via S. Quirico 19d, 16163 Genova, Italy.
}

\begin{abstract}
In recent years several legged/wheeled robots have been developed and proved their effective functionality in locomotion on uneven terrains. Many robotics researchers have been focusing on improving the locomotion speed, as well as the stability and robustness of such robots. High-speed locomotion of robots is however subject to various design challenges, especially in the development of actuators. The robotic applications which require high-speed motion in high torque operations along with the ability to manage dynamic physical interactions are not satisfied by the conventional robotics actuators deploying high reduction gearings. In this work, we present a quasi-direct drive actuator designed for continuous high-speed motions in high torque such as wheeled motions in mobile robot or joint motion in dynamic legged robots. The presented actuator exploits low reduction gearing so that it can render over $26 \mathrm{Nm}$ continuous torque while the actuator speed can exceed $37 \mathrm{rad} / \mathrm{s}$. Such characteristics enable exhibiting dynamic motions and dealing with large external impacts. The selection of motor and design of the gearing unit was carried out iteratively so that commercial items with minimum customization are employed and the outer diameter of the motor and the gearbox can be matching. A single-level planetary gearbox is devised for the reduction unit to ensure highly back drivability and transparency of the actuator thereby making the actuator robust against external impacts and allowing for accurate torque control using motor current measurement. The gear set design was carried out based on the AGMA gear torque calculation. Given the radial space required for the gearbox dealing with the torque requirements, the actuator motor was chosen to be small in height (pancake type), which ensures high torque density within smaller dimensions at high-speed operation. The mechanical design of the actuator is presented in this paper and the actuator specifications in terms of size and performance are compared with similar state-of-the-art actuators.
\end{abstract}

Keywords: Actuator; Direct Drive; Back Drivable

\section{Introduction}

The development of legged/wheeled robots allowed exploring external environments for applications such as urban search and rescue. Such applications often include maneuvers in rough and uneven terrains that are subject to unexpected interactions and abrupt impacts. To account for different performance criteria including mechanical resilience and energy efficiency, the development of robotics actuators introduces problems [1]. Conventional geared actuators, that are also commercially available, have managed to provide high torque density and control bandwidth, but only at the cost of significant friction and increased reflected inertia. While strain-wave gearing solutions e.g. harmonic drives significantly reduce the actuator mass and the friction, to improve the torque density and enable back-drivability, they present unwanted flexibility and are vulnerable to external impacts. 

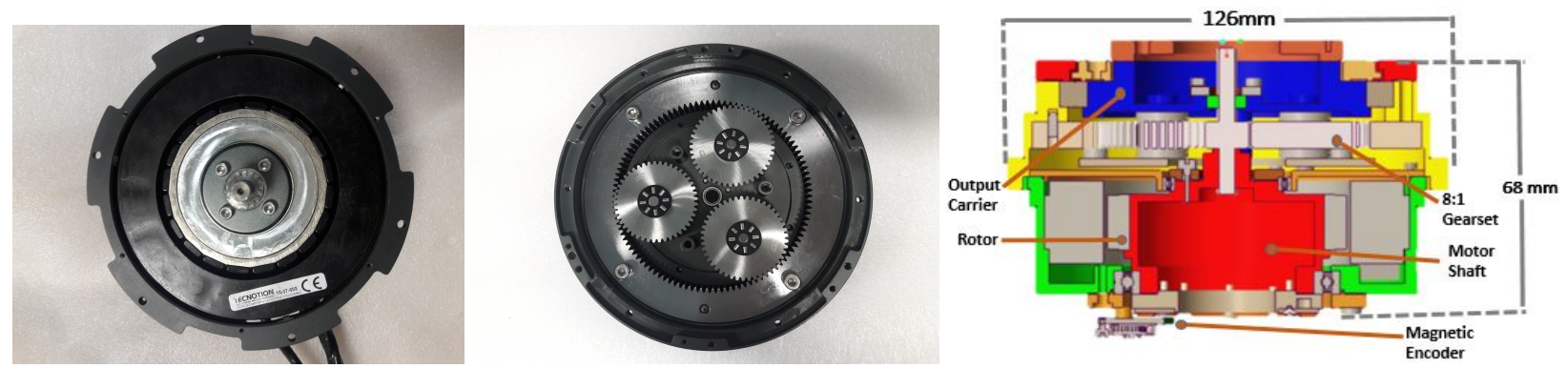

Figure 1. Figures from left: 1) The motor sub-assembly; 2) The gearbox sub-assembly; 3) The cross-section view of the actuator assembly CAD, fitting into $126 \times 68 \mathrm{~mm}$ dimension.

A set of state-of-the-art actuators, known as series elastic actuators (SEAs), see e.g. [2-4], have to compromise bandwidth to obtain compliance enabling harsh and/or unwanted interactions, in addition to other advantages such as energy efficiency in resonance motions. Several variations of SEAs have been developed to permit controlling the intrinsic stiffness (see e.g. [5]) and damping (see e.g. [6]), or to grant the replication of user-designed nonlinear stiffness and damping profiles (see e.g. [7]). Despite the benefits introduced by the intrinsic compliance, these actuators integrate additional components which increase the system complexity and mass, thereby resulting in limited benefits, especially for dynamic locomotion. Direct drive actuators $[8,9]$ on the other hand do not use gear reduction, thus eliminating the problems associated with gearboxes. They introduce high force transparency and are mechanically robust. However, most of these actuators are unable to provide a sufficiently large torque for common robotics applications when mass limitations are taken into account.

To address the limitations of direct drive units and high reduction geared actuators, quasi direct drives have been developed to generate sufficiently large torques required for a given application, without sacrificing back-drivability and/or mechanical resilience. These drives are often composed of torque motors (in contrast to power/speed motors) and single-stage gearing transmission, so that the output torque is amplified to some extent and intrinsic back-drivability is achieved, without compromising the system bandwidth. Moreover, while torque sensors (such as [10]) are an essential part of the state-of-the-art robotic platforms actuators e.g. [11], the low reduction of the gearing and the resulting high efficiency allows the motor torque, that can be realized by the current measurement, to be directly a valid indication of the output torque. Such a design provides an actuation solution for robots rendering dynamic motions such as jumping, as presented in MIT cheetah robot [12-14]. A similar customized quasi direct drive actuator was developed for the mini cheetah robot [15] that operates at lower torques. Yu et al. [16] presented the BIRO actuator, based on a high torque density customized motor, to develop a quasi direct drive for an exoskeleton with a peak torque of $42 \mathrm{Nm}$; however, the maximum speed it can reach is about $19 \mathrm{rad} / \mathrm{s}$. The Stanford Doggo [17], a light-weight jumping robot, uses an out-runner motor with a fairly low reduction to render considerably fast motion at the cost of low torque. Table. 2 details the available specifications of these actuators as compared to that of the proposed actuator.

This work introduces an actuator design for a new legged/wheeled hybrid robot, specifically targeted to perform fast and explosive motions while being also capable of supporting heavy-load applications. The article presents the details of the mechanical design of the actuator, relying upon commercial/datasheet components, and therefore limiting the customization and fabrications of mechanical parts to the corresponding housing and support parts, so that we can achieve a more economical, yet reliable, solution, which is tailored to the requirement of our target platform.

\section{Actuator Design}

The selection of components was carried out through an iterative process on the basis of the minimum torque and speed requirements determined from the specifications of the target platform, 
while we designed the gearbox to include only one stage reduction. The target continuous and peak output torques of the actuator are 20 and $40 \mathrm{Nm}$, respectively, and the target no-load speed is selected as $20 \mathrm{rad} / \mathrm{s}$. The motor and gearbox selection was therefore carried out in such a way that the outer diameter of the motor and the gearbox are as close as possible to each other so that the outer envelope of the actuator can be fairly continuous. The selected frameless motor is the Tecnotion QTR 105-25Z, which is a flat (pancake type) motor that can generate $3.3 \mathrm{Nm}$ continuous torque, while its peak torque can reach up to $6.9 \mathrm{Nm}$ and its speed can exceed $2700 \mathrm{rpm}$. It generates the target torques with a reduction ratio of $8: 1$, even when a fairly conservative overall efficiency of $80 \%$ is accounted for, while the no-load speed will be significantly higher than the target maximum speed.

The actuator assembly is shown in Fig. 1 consists two sub-assemblies: the motor and gearbox. The motor sub-assembly is shown on the left, which consists of the frameless motor and the corresponding parts including the stator housing, and the rotor shaft on which the gearbox input pinion is also assembled. The gearbox sub-assembly is shown in the middle of the Fig. 1, that includes the planet gears and their supporting carrier, as well as the ring gear. The gears are made of steel molybdenum alloy material produced by Kyouiku Gears. Table 1 details the specifications of the selected gears including torque rating. The maximum teeth strength of the gears was studied, the AGMA gear torque calculation formula [18] was used to ensure correct selection of the thickness, module, and material of the gears. Considering shape, geometry, and load factors specific to the gear, the maximum strength of gear teeth and the corresponding torque per tooth $T_{g}$ are calculated by (1) and (2) respectively,

$$
\begin{gathered}
W_{t}=\sigma K_{v} J m F / K_{m}, \\
T_{g}=P_{d} W_{t} / 2,
\end{gathered}
$$

where $W_{t}$ symbolizes the maximum shear strength of gear teeth, $\sigma$ is the allowable tooth root bending stress, $K_{v}$ and $J$ are the dynamic and geometry factors of the teeth, $m$ is the gear teeth module, $F$ is Face width, $K_{m}$ is the load distribution factor, and $P_{d}$ is the pitch diameter of the gear.

An important step in the planetary gear design is to specify the number of planet gears. While increasing the number of planet gears reduces the load per tooth, the maximum number of planet gears is often limited by space and geometry. Moreover, the addition of planet gears also escalates the overall cost and sound noise, and it is therefore desired to employ the minimum number of planet gears required to mesh with the pinion gear (sun), which is attached to the motor shaft to support the ultimate stall torque of the motor. Table. 1 also reports the maximum torque that can be transferred through the gears, per tooth, when utilizing three planet gears; as compared to the maximum admissible torque on the basis of the gear tooth shear strength extracted from (1). The maximum torque that can be applied to the pinion is set based on the ultimate torque of the motor, that leads to $\frac{6.9}{3}=2.3 \mathrm{Nm}$ torque per teeth when using three planet gears; while the torques applied to the planet and ring gears are derived using the statics analysis of the gears.

The total weight of the proposed actuator is about $1.9 \mathrm{Kg}$, and generates output continuous and peak torques of about $26.4 \mathrm{Nm}$ and $55.2 \mathrm{Nm}$ without accounting the for losses. The maximum speed reaches $37.2 \mathrm{rad} / \mathrm{s}$ with $48 \mathrm{~V}$ bus voltage, when only quadrature axis current is applied, while the implementation of field-weakening controllers can boost maximum velocity, see e.g. $[19,20]$. To this end, the rotor motion is measured using a 19-bit AkSim magnetic encoder, thereby allowing for

Table 1. Maximum torque that can be provided by the gears.

\begin{tabular}{|l|l|l|l|l|l|l|l|l|}
\hline Gears & $\begin{array}{l}\text { Number } \\
\text { of teeth }\end{array}$ & $\begin{array}{l}\text { Pitch } \\
\text { Diameter }\end{array}$ & Module & $\begin{array}{l}\text { Load Dist. } \\
\text { Factor }\end{array}$ & $\begin{array}{l}\text { Dynamic } \\
\text { Factor }\end{array}$ & $\begin{array}{l}\text { Geometry } \\
\text { Factor }\end{array}$ & $\begin{array}{l}\text { Max admissible } \\
\text { torque per tooth }\end{array}$ & $\begin{array}{l}\text { Max torque } \\
\text { applied per tooth }\end{array}$ \\
\hline Pinion & 15 & 12 & 0.8 & 1.3 & 0.75 & 0.29 & 2.34 & 2.3 \\
\hline Planets & 45 & 36 & 0.8 & 1.3 & 0.74 & 0.35 & 8.49 & 6.9 \\
\hline Ring & 105 & 84 & 0.8 & 1.3 & 0.8 & 0.42 & 17.36 & 16.1 \\
\hline
\end{tabular}


Table 2. The Specifications of the proposed actuators as compared to state-of-the-art quasi direct drives.

\begin{tabular}{|l|l|l|l|l|l|l|}
\hline Actuator & $\begin{array}{l}\text { Dimension } \\
(\mathrm{mm})\end{array}$ & $\begin{array}{l}\text { Peak Torque } \\
(\mathrm{Nm})\end{array}$ & $\begin{array}{l}\text { Nominal } \\
\text { Torque }(\mathrm{Nm})\end{array}$ & $\begin{array}{l}\text { Maximum } \\
\text { Speed }(\mathrm{rad} / \mathrm{s})\end{array}$ & $\begin{array}{l}\text { Peak torque density } \times \\
\text { maximum speed }(\mathrm{W} / \mathrm{kg})\end{array}$ & $\begin{array}{l}\text { Weight } \\
(\mathrm{Kg})\end{array}$ \\
\hline MIT Cheetah 1 & $\mathrm{N} / \mathrm{A}$ & 58 & $\mathrm{~N} / \mathrm{A}$ & $6.9^{*}$ & 219.29 & 1.825 \\
\hline Mini Cheetah & $96 \times 40$ & 17 & 6.9 & 40 & 1360 & 0.5 \\
\hline BIRO & $110 \times 51$ & 36 & 17.1 & 19 & 876.9 & 0.78 \\
\hline $\begin{array}{l}\text { Stanford } \\
\text { Doggo }\end{array}$ & $\mathrm{N} / \mathrm{A}$ & 4.8 & 1.51 & 251 & 3675 & 0.28 \\
\hline $\begin{array}{l}\text { Proposed } \\
\text { actuator }\end{array}$ & $126 \times 68$ & 55.2 & 26.4 & 37.2 & 1086.47 & 1.89 \\
\hline
\end{tabular}

${ }^{*}$ The value was computed using the corresponding motor torque constant $0.4 \mathrm{Nm} / \mathrm{A}$ and the supply voltage $100 \mathrm{~V}$, and considering the reduction ratio of 1:5.8, as reported in [12].

straightforward field-oriented current control. The lower-level controller is implemented on a custom electronics, where the innermost current loop is executed at $20 \mathrm{kHz}$, and the outer velocity loop is run at $2 \mathrm{kHz}$. The communication of the higher-level interface and the lower-level is carried out through the EtherCAT communication protocol.

Table. 2 presents the comparisons of some of the state-of-the-art quasi direct drive actuators with the presented actuator. It shows the proposed unit relying mainly upon commercial components (motor, gears, bearings, and other critical components) can exhibit notable characteristics. To present a quantifiable comparison, we defined an index based on the peak torque density and maximum reachable speed. By comparing the two drives proposed by the MIT group, we can see this power-density-like index drops about six times when the peak torque increases around three times. It shows our proposed unit renders an index about five times that of the MIT cheetah while generating a fairly similar peak torque, and renders a fairly higher power-density-like index than BIRO actuator while the proposed drive can generate $50 \%$ higher peak torque.

\section{Experimental Results}

To validate the performance of the actuator, specifically in terms of peak speed and continuous torque, two experiments were performed when the drive was velocity controlled:

1. Nominal Torque Evaluation: The actuator output shaft was attached to a lever, with a set of loads weighing about $14 \mathrm{Kg}$ hanging at a $20 \mathrm{~cm}$ distance with respect to the joint axis, replicating an external torque of approximately $28 \mathrm{Nm}$ which is mildly higher than the continuous torque of the actuator. A set of velocity references were commanded until the lever reaches the horizontal position, and it is held in that condition. The time history of current associated with this test is demonstrated in Fig. 2.

2. Maximum Speed Evaluation: The actuator output is free, and a few direct current commands are set to render the maximum velocity with the actuator, which is about $37 \mathrm{rad} / \mathrm{s}$ when taking about $4 \mathrm{~A}$ to overcome the frictional and other losses. The corresponding time history of current and velocity is illustrated in Fig. 3.

\section{Conclusion}

This article introduces the design of a new quasi direct drive actuator, on the basis of a high torque density motor and a low reduction-ratio gearing transmission. The proposed design was discussed and the actuator was successfully developed. Experimental results validate the target characteristics of the actuator in terms of torque and speed, which presents the potentiality of the proposed drive in empowering a platform that can perform dynamic motions and can exhibit high strength. 


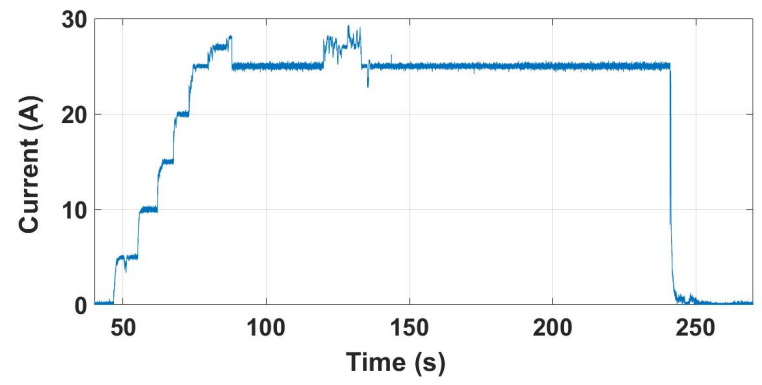

Figure 2. Time history of current when executing a torque of $28 \mathrm{Nm}$.
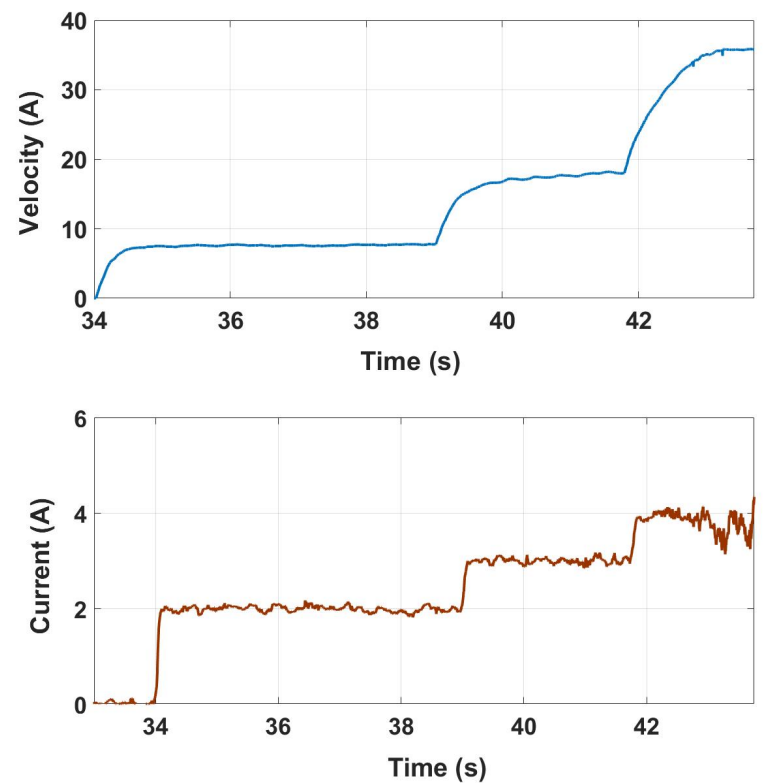

Figure 3. Time history of current and velocity when moving with no load.

\section{References}

1. Kashiri, N.; Abate, A.; Abram, S.J.; Albu-Schaffer, A.; Clary, P.J.; Daley, M.; Faraji, S.; Furnemont, R.; Garabini, M.; Geyer, H.; others. An overview on principles for energy efficient robot locomotion. Frontiers in Robotics and AI 2018, 5, 129.

2. Pratt, G.A.; Williamson, M.M. Series elastic actuators. IEEE/RSJ International Conference on Intelligent Robots and Systems, 1995, Vol. 1, pp. 399-406.

3. Tsagarakis, N.G.; Laffranchi, M.; Vanderborght, B.; Caldwell, D.G. A compact soft actuator unit for small scale human friendly robots. IEEE international conference on robotics and automation, 2009, pp. 4356-4362.

4. Hutter, M.; Remy, C.D.; Hoepflinger, M.A.; Siegwart, R. Scarleth: Design and control of a planar running robot. IEEE/RSJ International Conference on Intelligent Robots and Systems, 2011, pp. 562-567.

5. Grioli, G.; Wolf, S.; Garabini, M.; Catalano, M.; Burdet, E.; Caldwell, D.; Carloni, R.; Friedl, W.; Grebenstein, M.; Laffranchi, M.; others. Variable stiffness actuators: The user's point of view. The International Journal of Robotics Research 2015, 34, 727-743.

6. Laffranchi, M.; Chen, L.; Kashiri, N.; Lee, J.; Tsagarakis, N.G.; Caldwell, D.G. Development and control of a series elastic actuator equipped with a semi active friction damper for human friendly robots. Robotics and Autonomous Systems 2014, 62, 1827-1836.

7. Kashiri, N.; Caldwell, D.G.; Tsagarakis, N. A self-adaptive variable impedance actuator based on intrinsic non-linear compliance and damping principles. 2017 IEEE International Conference on Robotics and Automation (ICRA). IEEE, 2017, pp. 1248-1254.

8. Asada, H.; Youcef-Toumi, K. Direct-drive Robots: Theory and Practice; MIT Press, 1987. 
9. Carignan, C.R.; Cleary, K.R. Closed-loop force control for haptic simulation of virtual environments 2000. pp. 1-14.

10. Kashiri, N.; Malzahn, J.; Tsagarakis, N.G. On the sensor design of torque controlled actuators: A comparison study of strain gauge and encoder-based principles. IEEE Robotics and Automation Letters 2017, 2, 1186-1194.

11. Kashiri, N.; Baccelliere, L.; Muratore, L.; Laurenzi, A.; Ren, Z.; Hoffman, E.M.; Kamedula, M.; Rigano, G.F.; Malzahn, J.; Cordasco, S.; others. CENTAURO: A hybrid locomotion and high power resilient manipulation platform. IEEE Robotics and Automation Letters 2019, 4, 1595-1602.

12. Seok, S.; Wang, A.; Chuah, M.Y.; Hyun, D.J.; Lee, J.; Otten, D.M.; Lang, J.H.; Kim, S. Design principles for energy-efficient legged locomotion and implementation on the MIT cheetah robot. IEEE/ASME transactions on mechatronics 2014, 20, 1117-1129.

13. Wensing, P.M.; Wang, A.; Seok, S.; Otten, D.; Lang, J.; Kim, S. Proprioceptive actuator design in the MIT Cheetah: Impact mitigation and high-bandwidth physical interaction for dynamic legged robots. IEEE transactions on robotics 2017, 33, 509-522.

14. Ding, Y.; Park, H.W. Design and experimental implementation of a quasi-direct-drive leg for optimized jumping. 2017 IEEE/RSJ International Conference on Intelligent Robots and Systems (IROS). IEEE, 2017, pp. 300-305.

15. Katz, B.; Di Carlo, J.; Kim, S. Mini cheetah: A platform for pushing the limits of dynamic quadruped control. IEEE International Conference on Robotics and Automation, 2019, pp. 6295-6301.

16. Yu, S.; Huang, T.H.; Yang, X.; Jiao, C.; Yang, J.; Chen, Y.; Yi, J.; Su, H. Quasi-Direct Drive Actuation for a Lightweight Hip Exoskeleton with High Backdrivability and High Bandwidth. IEEE/ASME Transactions on Mechatronics 2020.

17. Kau, N.; Schultz, A.; Ferrante, N.; Slade, P. Stanford doggo: An open-source, quasi-direct-drive quadruped. IEEE International Conference on Robotics and Automation, 2019, pp. 6309-6315.

18. Association, A.G.M.; Institute, A.N.S. Fundamental Rating Factors and Calculation Methods for Involute Spur and Helical Gear Teeth; AGMA standard, American Gear Manufacturers Association, 2004.

19. Roozing, W.; Kashiri, N.; Tsagarakis, N.G. Enhanced explosive motion for torque controlled actuators through field weakening control. 2018 IEEE/RSJ International Conference on Intelligent Robots and Systems (IROS). IEEE, 2018, pp. 1-8.

20. Mohammadnia, M.; Kashiri, N.; Braghin, F.; Tsagarakis, N.G. Flux Regulation for Torque-controlled Robotics Actuators. 2019 19th International Conference on Advanced Robotics (ICAR). IEEE, 2019, pp. 93-98.

(C) 2020 by the authors. Submitted to Journal Not Specified for possible open access publication under the terms and conditions of the Creative Commons Attribution (CC BY) license (http://creativecommons.org/licenses/by/4.0/). 